JOURNAL OF THEORETICAL

AND APPLIED MECHANICS

59, 1, pp. 135-142, Warsaw 2021

https://doi.org/10.15632/jtam-pl/130454

\title{
LIQUID BRIDGE IN SLIT PORE GEOMETRY
}

\author{
Junhui Hu, Menguiao Wu, Kezhao Bai, Li Yang \\ College of Physics Science and Technology, Guangxi Normal University, Guilin, China \\ e-mail:yangli@mailbox.gxnu.edu.cn
}

\begin{abstract}
In this work, the morphology of a liquid bridge in a slit pore geometry was investigated as a function of both the bridge height and aspect ratio (height/width). The end contour interface of the liquid bridge was modeled by using a saddle shape, and the liquid-air interface was described via an arc of a circle. By employing the free energy approach, a simple formula was obtained to predict variation of the pinning angle as a function of the distance between the slits. The pinning angle depended on the liquid volume and on both the wetting properties and the geometry of the system (height and width). The critical aspect ratio at which the liquid bridge meniscus transitioned from concave to convex was determined. The calculations were in good agreement with the experimental data. The morphology of the liquid bridges in a slit pore geometry can be used in various fields such as the packaging of electronic and micro-electromechanical systems.
\end{abstract}

Keywords: liquid bridge, slit, pinning angle, critical aspect ratio, saddle shape

\section{Introduction}

Investigations on the morphology of liquid bridges created between two rectangular structured surfaces provide a theoretical basis for the development of electronic packaging processes (Chiang end Chen, 1998), high-density packaging (Chen et al., 2005), self-assembly processes (Xu et al., 2006) and micro-electromechanical systems (Raccurt et al., 2004). For instance, the liquid (or fluid) plays a significant role in formation of liquid bridges and in delamination processes during soldering of a quad flat non-leaded package onto a printed circuit board. In an environment with a certain humidity level, the presence of a liquid bridge affects friction, adhesion and energy dissipation. For this reason, it has a significant effect on micro electromechanical systems (MEMS) and atomic force microscopy systems (Wei and Zhao, 2007). Therefore, it is very important and necessary to fully understand the liquid bridge morphology between two parallel surfaces.

Several studies have considered the contribution of the wetting and shape of an axisymmetric liquid bridge formed across two flat surfaces (Evans et al., 1986; Swain and Lipowsky, 2000; Valencia et al., 2001; Peng and Li, 2007; Broesch and Frechette, 2012; Broesch et al., 2013; Zhu et al., 2015). For instance, Swain and Lipowski (2000) investigated wetting phenomena occurring between one pair of opposite stripes. A bridge can span from a lyophilic stripe belonging to a surface to another one situated on the opposite surface. This generates a force which acts between the substrates and can be evaluated via 2D analysis. Valencia et al. (2001) investigated morphology of anvil-like shaped liquid bridge phases within a series of slit pores with a hydrophilic strip. They used a lattice gas model and minimization methods to simulate the mean curvature radius of the liquid-vapor interface. Broesch and Frechette (2012) performed several experiments and a series of surface evolver simulations to characterize morphology of liquid bridges confined within a slit pore of variable height. Their results show that the pinning angle increases with an increase in the pore height. This is associated with transition in the mean curvature radius of the 
bridge from concave (negative) to convex (positive). Based on three simple geometric arguments (Broesch et al., 2013), the authors developed a simple formula to predict the pinning angle solely based on the contact angle, height and width of the bridge. The experimental results reported in their paper showed that the pinning angle was closely related to the volume. Zhu et al. (2015) solved differential equations which clarify the relation between capillary forces and the Laplace pressure of the capillary bridge as a function of the shape during the bridge stretching process. However, their simulation results have not been experimentally verified.

Previous studies aimed at predicting morphology and capillary forces generated by liquid bridges via surface evolver simulations (Broesch and Frechette, 2012; Broesch et al., 2013; Zhu et al., 2015; Peng and Li, 2007; Petkov and Radoev, 2014; Princen, 1970). However, few theoretical calculation methods are available to describe the liquid bridges between two slits since the analytical description of the menisci at the two terminals remains challenging. Moreover, the relation between the pinning angle and the volume still has to be clarified. In this paper, the morphology of liquid bridges confined within a slit pore was investigated via an elliptic approximation. A relation between the pinning angle and properties of the strip (wetting, height, width, and volume) was proposed. Furthermore, the critical aspect ratio (height/width) at which the liquid bridge meniscus transitions from concave to convex was obtained. These results provide the curvature radius of the liquid bridge terminals and the ratio between the volume at both ends of the liquid bridge and the total volume. The results of the theoretical model are in close agreement with the experimental data from the previous work.

\section{Analytical modeling}

In this work, a liquid bridge in a slit consisting of two rectangular flat and parallel plates separated by a distance $H$ (Fig. 1) is considered. The model is based on three assumptions for the geometry of the liquid bridge:

- The liquid-air interface can be described by using arcs of circles.

- The liquid at two terminals can be described by a saddle shape, and the cross section along the horizontal direction can be approximated with an ellipse. The triple contact line has a fixed plate width but is free to advance or recede along its length.

- Following the Princen (1970) approach, the contribution of surface energy at the two terminals is neglected.

In Fig. 1, the arc $A B C$ is a contour of the middle of the liquid bridge, the arc $A D C$ is a contour of the liquid-solid interface. Black represents slits and blue represents liquid bridge, $L$ is length of the liquid-solid interface, $L_{x}$ - length of the middle section of the liquid bridge, $W$ - plate width, $R_{n}$ - length in the $x$ direction of the saddle-shaped neck, $r_{d}$ corresponds to the radius of the meniscus at both ends, $\theta$ is the contact angle, $x_{m}, y_{m}$ are the coordinates of $M$ and $R_{1}$ is the radius of the thinnest part on the side of the liquid bridge.

Based on the Young-Laplace equation (Petkov and Radoev, 2014), the pressure difference between the inside and outside part of the system at the liquid-air interface (i.e. the meniscus) can be written as follows

$$
\Delta p_{\text {end }}=-\gamma\left(\frac{2 \cos \theta}{H}-\frac{R_{n}}{\left(\frac{W}{2}-H \frac{1-\sin \alpha}{2 \cos \alpha}\right)^{2}}\right) \quad \Delta p_{\text {side }}=-\frac{2 \gamma \cos \alpha}{H}
$$

Here, $\Delta p$ corresponds to the Laplace pressure, $\alpha$ is the pinning angle, and $\theta$ is the contact angle. $R_{n}$ represents the length along the $x$ direction of the saddle-shaped neck. Since the mean 
(a)

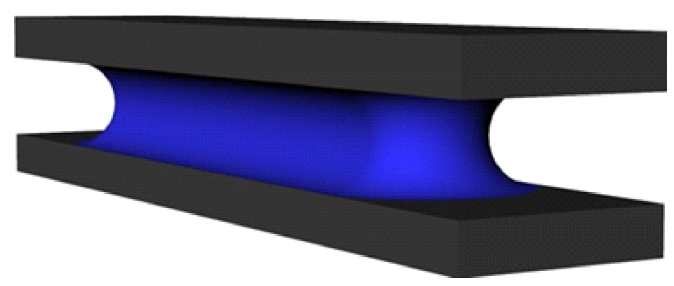

(c)

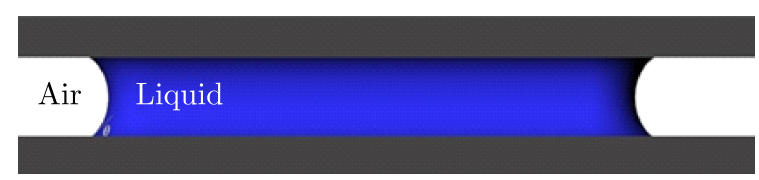

(e)

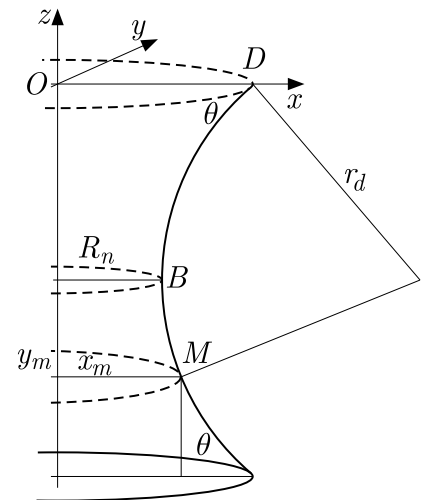

(b)

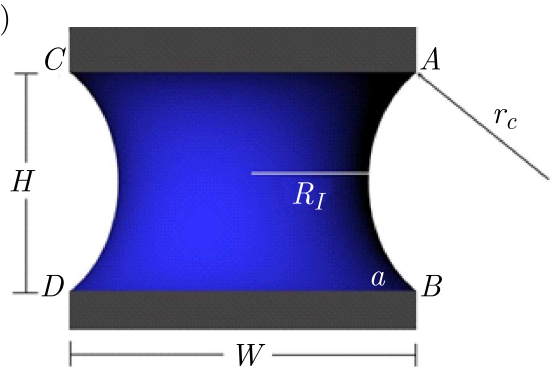

(d)

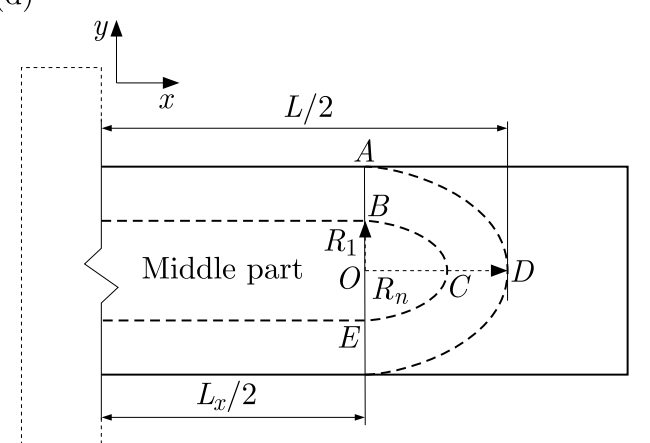

Fig. 1. Liquid bridge slit pore geometry: (a) three quarters view, (b) side view, (c) front view, (d) top view of its right end, (e) side view of the end

curvature is identical for all the points along the liquid bridge profile, the relation between $\alpha$ and $\theta$ can be defined as follows

$$
\cos \theta=\cos \alpha+\frac{H R_{n}}{2\left(\frac{W}{2}-H \frac{1-\sin \alpha}{2 \cos \alpha}\right)^{2}}
$$

The $\alpha$ value can be predicted without defining $R_{n}$, which is closely related to the volume of the liquid bridge. The volume is calculated as follows (details are given in Supporting Information Appendix)

$$
\begin{aligned}
V= & \left(L-2 R_{n}-H \frac{1-\sin \theta}{\cos \theta}\right)\left(W H-\frac{H^{2}}{4} \frac{\frac{\pi}{2}-\alpha-\cos \alpha \sin \alpha}{\cos ^{2} \alpha}\right) \\
& +\frac{\pi W}{2}\left[R_{n} H \frac{\pi-2 \theta}{2 \cos \theta}+\frac{H^{2}}{4 \cos ^{2} \theta}(\pi-2 \theta-2 \cos \theta)\right]
\end{aligned}
$$

To obtain the unknown parameters, the value of $L$ is the key parameter to define the shape of the liquid bridge. Since the contribution of two ends is relatively small with respect to the middle part, it is assumed that the liquid in the middle part grows or shrinks in length while the cross-section is constant. The lengthening of the liquid bridge results in the wetting of an area $(A C+B D) d L_{x}$ of the originally nonwetted plates and in the creation of the area $(A B+C D) d L_{x}$ of the liquid-air surface (Fig. 1b). The variation of the free energy can be expressed as follows

$$
d E=\left[(A C+B D)\left(\gamma_{S L}-\gamma_{S A}\right)+(A B+C D) \gamma_{L A}\right] d L_{x}
$$


where $\gamma_{S L}, \gamma_{S A}$ and $\gamma_{L A}(=\gamma)$ are the solid-liquid, solid-air and liquid-air interfacial tensions, respectively. $A B$ and $C D$ are the liquid-air surfaces and $A C$ and $B D$ are the liquid-solid surfaces. According to the Young-Dupré equation

$$
\gamma_{S A}-\gamma_{S L}=\gamma \cos \theta
$$

Then, Eq. (2.4) can be written in the form

$$
d E=[(A B+C D)-(A C+B D) \cos \theta] \gamma d L_{x}
$$

From Fig. 1b, we get $A B=C D=2(\pi / 2-\alpha) r_{c}$ and $A C=B D=W$. Then, the free surface energy change is

$$
d E=\left[2 r_{c}(\pi-2 \alpha)-2 W \cos \theta\right] \gamma d L_{x}
$$

The variable $r_{c}$ corresponds to the radius of the meniscus at both sides, and can be defined as follows

$$
r_{c}=\frac{H}{2 \cos \alpha}
$$

The work done on the liquid bridge $d W_{L}$ results from the pressure difference between the atmosphere and the liquid

$$
d W_{L}=\Delta p_{\text {side }} d V_{\text {middle }}
$$

$V_{\text {middle }}$ is the volume of the middle part of the liquid bridge (excluding saddle-shaped liquid volumes at both ends). For the middle part of the liquid bridge, the variation of the energy associated with the volume is equal to the variation of the work. First, Eq. (2.8) is substituted into Eq. (2.7), and then Eq. (2.7) and Eq. (2.9) are made equal $d E=d W_{L}$. Finally, the following equation is obtained

$$
L_{x}=\frac{2 V \cos \alpha}{H\left(2 W \cos \theta-H \frac{\pi-2 \alpha}{\cos \alpha}\right)}
$$

Then $L$ can be expressed as follows

$$
L=L_{x}+2 R_{n}+H \frac{1-\sin \theta}{\cos \theta}
$$

Substituting $R_{n}$ (Eq. (2.2)) and $L$ (Eq. $(2,11)$ ) into Eq. (2.3), $\alpha$ can be determined for a fixed value of $H, W, \theta$ and $V$. All simulations and calculations were performed with Matlab.

\section{Numerical solution}

\subsection{Influence of $H$ on the pinning angle}

The pinning angle as a function of height for different volumes $V$ and widths $W$ of the plate is shown in Fig. 2. When $H$ is small, the calculated pinning angles are almost the same under different contact angles. However, when $H$ is relatively large, the difference is obvious. The contact angle has a great effect on the result of the pinning angle when curvature of the liquid bridge is negative (the profile is convex).

The shape of the liquid bridges was characterized by considering the pinning angle $\alpha$, length $L$ and height $H$. With an increase in height of the plate, the liquid bridge shrinks along the plate 
(a)

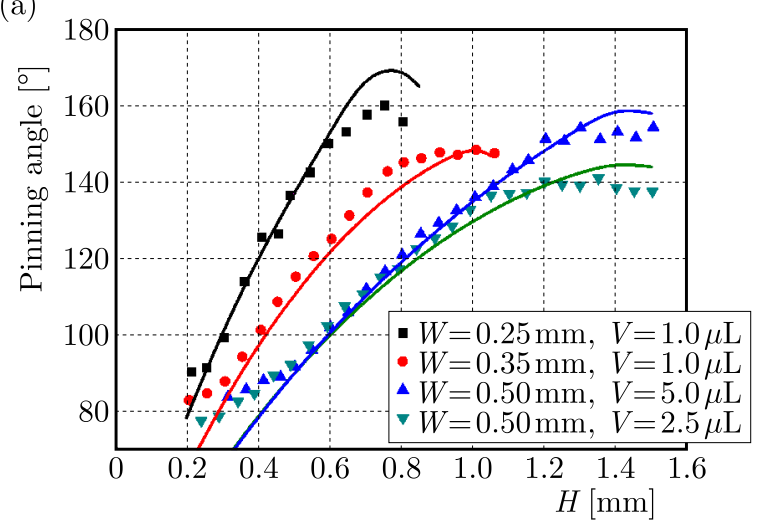

(b)

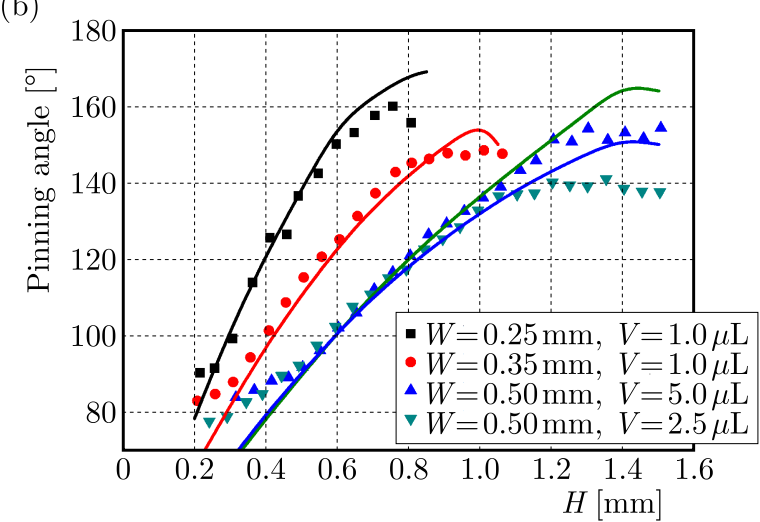

(c)

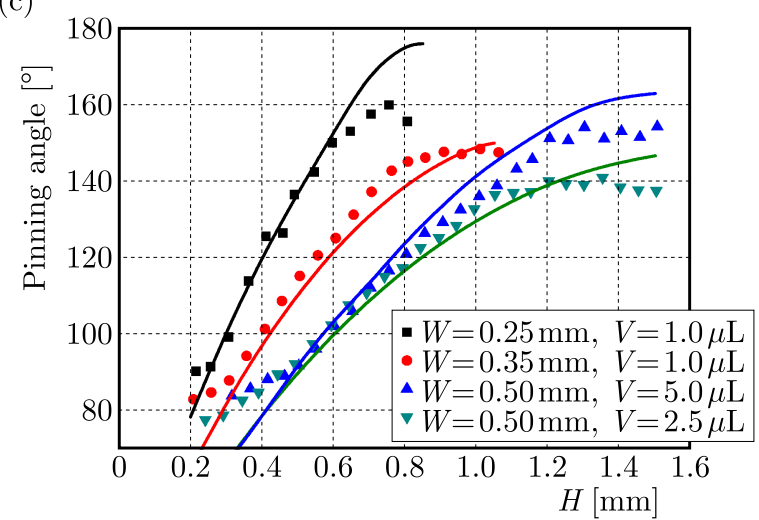

Fig. 2. Variation of the pinning angle as a function of height. The data points were obtained experimentally (Broesch and Frechette, 2012). In this case, the surface tension is $\gamma=51 \mathrm{mN} / \mathrm{m}$ for a water-glycerol liquid (Broesch and Frechette, 2012). The solid lines are the results of the simulation performed in this work considering: (a) $\theta=2^{\circ}$, (b) $\theta=10^{\circ}$, (c) $\theta=25^{\circ}$

surface, and the pinning angle increases. Moreover, the liquid bridge becomes thicker until it reaches its maximum. The simulation results of the pinning angles based on Eq. (2.2) are in good agreement with the experimental results. However, both high and low values of the height result in a less satisfactory agreement between the theoretical and experimental results. The findings suggest that this deviation arises from two causes: 1) The use of the circular arc approximation for the contour surfaces of the liquid bridge along its two sides, which implies that the surface tension effect dominates and that gravitation is negligible. Based on the results from previous work (Broesch et al., 2013), this approximation induces an error at this scale. 2) The contribution of the liquid volume at both ends is neglected.

\subsection{Influence of contact angle on $R_{1}$}

The calculation results satisfactorily predict the curvature change (from concave to convex in Fig. 3b) and the dependence on wetting properties. These results follow the same trends as the results obtained from Surface Evolver simulation by Broesch et al. (2013), see Fig. 5c.

\subsection{Influence of $H$ on $R_{1}$}

The evolution of $1 / R_{1}$ (obtained from Eq. (3.1)) as a function of the $H / W$ ratio is shown in Fig, 4

$$
R_{1}=\frac{W}{2}-\frac{H(1-\sin \alpha)}{2 \cos \alpha}
$$


(a)

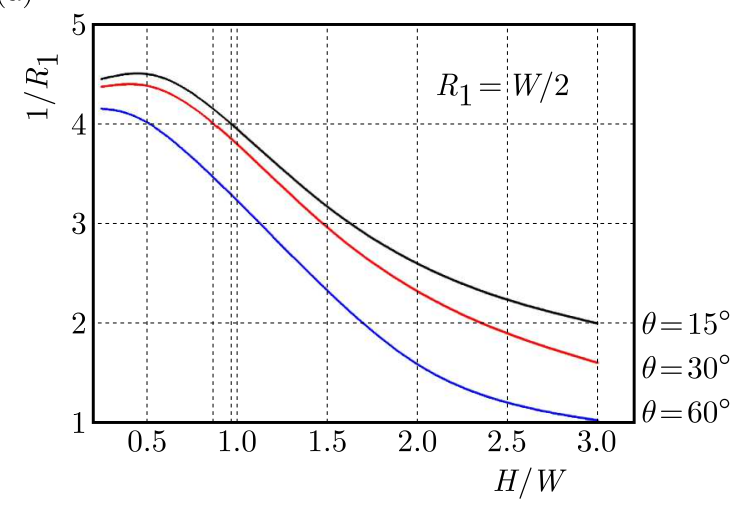

(b)

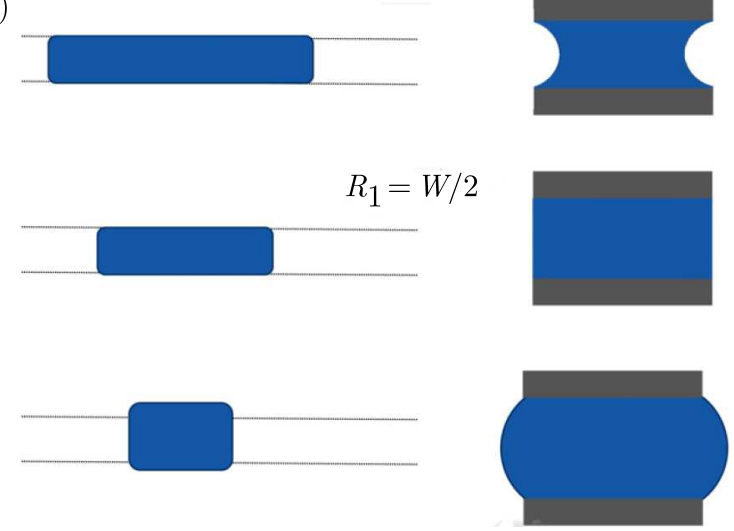

Fig. 3. (a) Evolution of $R_{1}$ for three different contact angles $W=0.5, V=2.5$ (Broesch et al., 2013). (b) Top view and front view of the liquid bridge

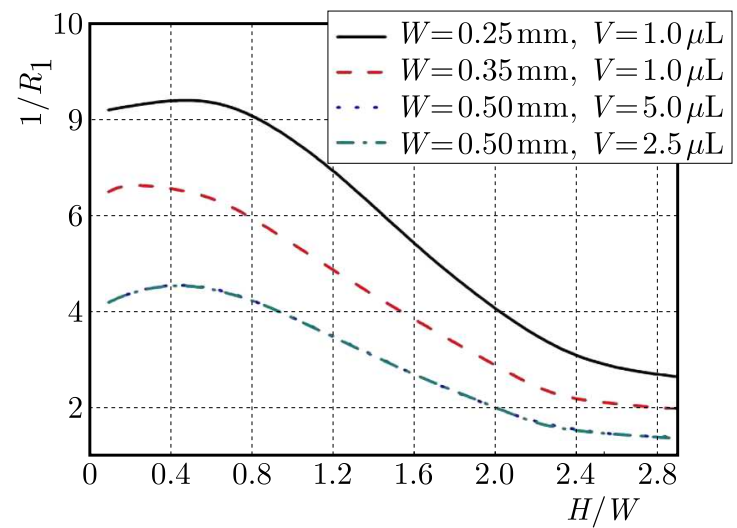

Fig. 4. Trend of $1 / R_{1}$ curve as a function of the aspect ratio $H / W$

The function reaches its maximum value and, then, it decreases. The trend of this curve is similar with that obtained via Surface Evolver simulations by Broesch (Fig. 5c) (Broesch et al., 2013). The results show that $R_{1}$ is not constant as a function of height, which cannot be approximated by $W / 2$. When $R_{1}=W / 2$ for $\alpha=90^{\circ}$, the Laplace pressure transitions from negative to positive values, and the liquid-air interface at both sides of the bridge changes from concave to convex (Galaktionov et al., 2017). The major curvature radius $r_{c}$ can then be approximated to infinity in Eq. (2.7), leading to

$$
[(A B+C D)-(A C+B D) \cos \theta]=2 H-2 W \cos \theta=0
$$

Therefore, we get

$$
\frac{H}{W}=\cos \theta
$$

For a fixed value of $\theta$, the critical aspect ratio (height/width) can be determined for $\alpha=90^{\circ}$. Equation (3.3) is identical to Eq. (2.5) obtained by Broesch et al. (2013) via an experimental approach $\left(\alpha=90^{\circ}\right)$.

\subsection{Influence of $H$ on $R_{n}$ and $V_{\text {end }}$}

In the energy balance equation, Eq. (2.7), the contribution of liquid volume at both ends is neglected assuming that its volume is small in comparison with the total liquid volume. Figure 5 shows $R_{n} / L$ and $V_{\text {end }} / V$ ratios as functions of $H$. For a fixed value of $W$, the smaller the volume 
is, the more prominent are the two bridge terminals (saddle shape). For a fixed volume, the larger is $W$, the more prominent the bridge terminals are. For a rather large value of the liquid bridge volume, $R_{n} / L$ remains relatively small. The energy of the liquid-air surface at both terminals is relatively flat and can be ignored. When volume of the liquid bridge is small, the energy at the two terminals cannot be ignored. Therefore, there is a discrepancy between the theoretical and experimental values arises.

(a)

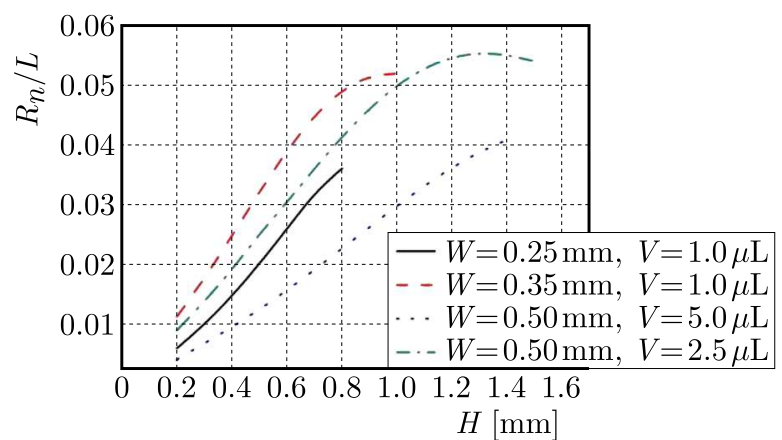

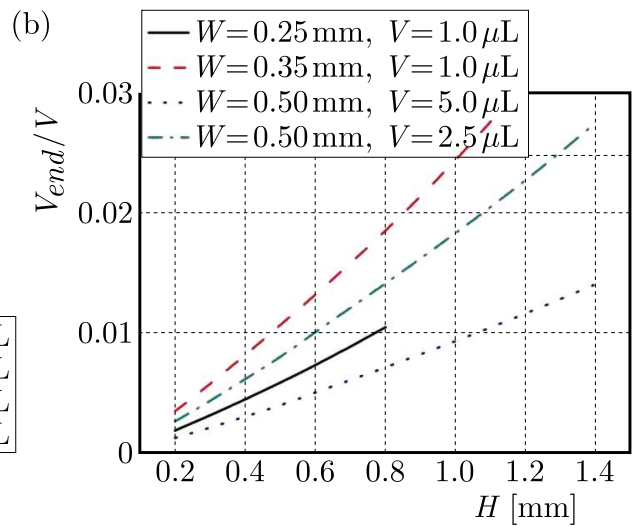

Fig. 5. (a) $R_{n} / L$ as a function of height. (b) $V_{\text {end }} / V$ as a function of height. $V_{\text {end }}$ corresponds to the saddle-shaped liquid volume at both terminals of the liquid bridge

\section{Conclusion}

In this paper, an analytical model is presented to describe the liquid bridge morphology in slit pore geometry. The results were confirmed by experimental data. The relation defining the effect of the pinning angle of the liquid bridge on the plate width and height as well as the liquid volume is derived. Furthermore, the critical aspect ratio (height/width) at which the liquid bridge meniscus transitions from concave to convex is determined for a fixed volume. Since the shape evolution of the liquid bridge is characterized by a high degree of complexity, further investigations are necessary to reveal the physics behind it.

\section{Acknowledgement}

This study was supported by the National Natural Science Foundation of China under Grant No. 11665007, the Guangxi Natural Science Foundation Program No. 2018GXNSFAA138190, the Guangxi province Higher Educational Science and Technology program No. 2018KY0085, and the Guangxi key research and development program under Grant No. AB18221033.

\section{References}

1. Broesch D.J., Dutka F., Frechette J., 2013, Curvature of capillary bridges as a competition between wetting and confinement, Langmuir, 29, 15558-15564

2. Broesch D.J., Frechette J., 2012, From concave to convex: capillary bridges in slit pore geometry, Langmuir, 28, 15548-15554

3. Chen W., Lin S., Chiang K., 2005, Stability of solder bridging for area array type packaging, Computers, Materials and Continua, 2, 3, 151-153

4. Chiang K.N., Chen W.L., 1998, Electronic packaging reflow shape prediction for the solder mask defined ball grid array, Journal of Electronic Packaging, 120, 175-178 
5. Evans R., Marconi U.M.B., Tarazona P., 1986, Capillary condensation and adsorption in cylindrical and slit-like pores, Journal of the Chemical Society, Faraday Transactions 2: Molecular and Chemical Physics, 82, 1763-1787

6. Galaktionov E.V., Galaktionova N.E., Tropp E.A., 2017, Shape of the surface of a vertical liquid bridge between two parallel solid planes taking into account the gravity force for small Bond numbers, Technical Physics, 62, 1482-1489

7. PEnG Y.F., Li G.X., 2007, An elastic adhesion model for contacting cylinder and perfectly wetted plane in the presence of meniscus, Journal of Tribology, 129, 231-234

8. Petkov P.V., Radoev B.P., 2014, Statics and dynamics of capillary bridges, Colloids and Surfaces A: Physicochemical and Engineering Aspects, 460, 18-27

9. Princen H.M., 1970, Capillary phenomena in assemblies of parallel cylinders: III. Liquid columns between horizontal parallel cylinders, Journal of Colloid and Interface Science, 34, 171-184

10. Raccurt O., Tardif F., D'Avitaya F.A., Vareine T., 2004, Influence of liquid surface tension on stiction of SOI MEMS, Journal of Micromechanics and Microengineering, 14, 1083-1085

11. Swain P.S., Lipowsky R., 2000, Wetting between structured surfaces: Liquid bridges and induced forces, Europhysics Letters, 49, 203-206

12. Valencia A., Brinkmann M., Lipowsky R., 2001, Liquid bridges in chemically structured slit pores, Langmuir, 17, 3390-3399

13. Wei Z., Zhao Y.P., 2007, Growth of liquid bridge in AFM, Journal of Physics D: Applied Physics, 40, 4368-4370

14. Xu J., Xia J., Hong S.W., Lin Z., Qiu F., YAng Y., 2006, Self-assembly of gradient concentric rings via solvent evaporation from a capillary bridge, Physical Review Letters, 96, 066104

15. Zhu Z.F., Jia J.Y., Fu H.Z., Chen Y.L., Zeng Z., Yu D.L., 2015, Shape and force analysis of capillary bridge between two slender structured surfaces, Mechanical Sciences, 6, 211-220 